\title{
Three-Dimensional Flow Visualization Using a Pulse Burst Laser System
}

\author{
Kyle Lynch ${ }^{*}$ and Dr. Brian S. Thurow ${ }^{\dagger}$ \\ Advanced Laser Diagnostics Laboratory \\ Auburn University, AL 36849
}

\begin{abstract}
Further development of a high-speed three-dimensional flow visualization system is explored. The technique is based on the high-speed scanning and imaging of a laser sheet produced by a pulse burst laser capable of operating in excess of $1 \mathrm{MHz}$. The focus of this study is on the further development of the pulse burst laser, synchronization between the multiple system components, and data processing to yield 3-D images and high-quality 2-D images. Multiple 3-D visualizations (220 x $220 \times 68$ voxel resolution) of an acousticallyexcited jet are presented, each taken at "full-speed," with the scanning mirror directing the laser through the flow, and the high-speed camera operating at 500,000 fps. In this configuration, each 3-D image took $136 \mu$ s to acquire. The presented visualizations demonstrate the ability of the technique to visualize complex, three-dimensional flow structures, such as ring vortices. Future work will concentrate on increasing laser intensity at shorter pulse durations to increase resolution and allow the technique to be used for supersonic flows.
\end{abstract}

\section{Introduction}

Techniques for non-invasive flow diagnostics using lasers have seen drastic improvements in speed and resolution in recent years, due to advances in CCD sensors and the demonstration of high-power laser pulse generation techniques, both operating in the $\mathrm{MHz}$ range. The combination of these advanced technologies allows improved study of many dynamic flow problems occurring at high speeds by implementing traditional particle image velocimetry (PIV) and other methods. In addition, the magnitude of these advances has made possible entirely new flow diagnostic techniques. Presented in this paper is a description of the final development and performance of a three-dimensional flow visualization system utilizing these advanced technologies. The imaging concept is based on scanning a high-power laser sheet through a flow, taking short, two-dimensional pictures during the scan, and reassembling the image sequence to create a three-dimensional image. Figure 1 presents a diagram of the concept from a top and side view.

This method for three-dimensional imaging has been demonstrated before for low speed flows where long camera exposures could capture an image without substantial distortion due to the movement of the flow. Longer exposure times also allowed continuous-wave and low energy pulsed laser sources to be used, which can provide sufficient energy for flow visualization using long exposure times. ${ }^{1}$ Expanding this technique to high speed flows requires ultra-high speed cameras capable of $100 \mathrm{~s}$ of $\mathrm{kHz}$ frame rates and exposure times on the order of $10 \mathrm{~s}$ of nanoseconds. Due to the shorter exposures, continuouswave laser sources can no longer provide sufficient energy for flow visualization, and more powerful pulsed laser systems must be devised. Therefore, a pulse burst laser has been developed. The system is a $3^{\text {rd }}$ generation design based on similar systems

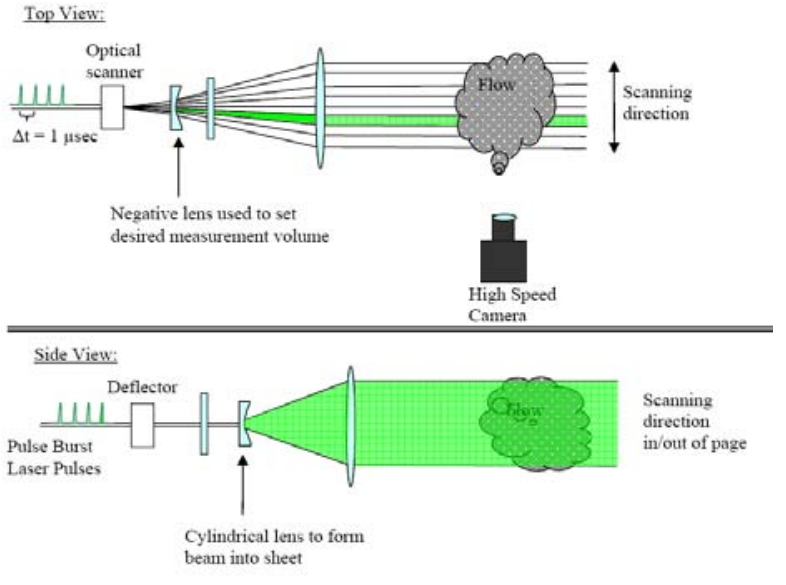

Figure 1: Diagram of 3-D Imaging Concept

\footnotetext{
* Undergraduate Research Assistant, Auburn University, AIAA Student Member

${ }^{\dagger}$ Assistant Professor, AIAA Faculty Advisor, AIAA Member
} 
described by Lempert et al. ${ }^{2}$ and Wu et al. ${ }^{3}$, and Thurow et al. ${ }^{4}$ The detailed design for pulse generation, slicing, and amplification has been reported before in Thurow and Satija. ${ }^{5}$ Due to difficulties with the amplifier power supply unit, the system has not been fully operational until recently. This paper reports the current performance of the pulse burst laser system, the integration effort of the laser and other systems to enable the high-speed 3-D imaging concept, and visualizations obtained using the method.

\section{Pulse Burst Laser System Completion}

An overview of the design of the pulse burst laser system is given here for informational purposes and to describe the actual implementation. The fundamental concept of operation for the laser system is to generate and amplify a train of short-duration laser pulses to high energies.

\section{A. Pulse Generation}

The initial laser energy is provided by a $100 \mathrm{~mW}$ continuous-wave Nd:YAG laser, with output at $1064 \mathrm{~nm}$. The output beam is focused into an Acousto-Optic Modulator (AOM), a device traditionally used in Q-switched lasers. By using compression waves propagating through a crystal to modulate the density (and subsequently the index of refraction), the AOM serves as a tunable diffraction grating which can be turned on and off to deflect the continuous-wave input at rates up to $100 \mathrm{MHz}$. The AOM used here has a measured deflection efficiency of $61 \%$. For three-dimensional visualization purposes, pulses of 40 to $300 \mathrm{~ns}$ duration (measured at full-width, halfmaximum, or FWHM) are created, resulting in individual pulse energies on the order of nanojoules to be used as input to the amplifier chain. An oscilloscope trace of one such pulse is shown in Figure 2, where a rise time on the order of $\sim 7 \mathrm{~ns}$ can be observed. Figure 3 shows a complete 68-pulse train of $60 \mathrm{~ns}$ pulses, occurring in $136 \mu \mathrm{s}$.

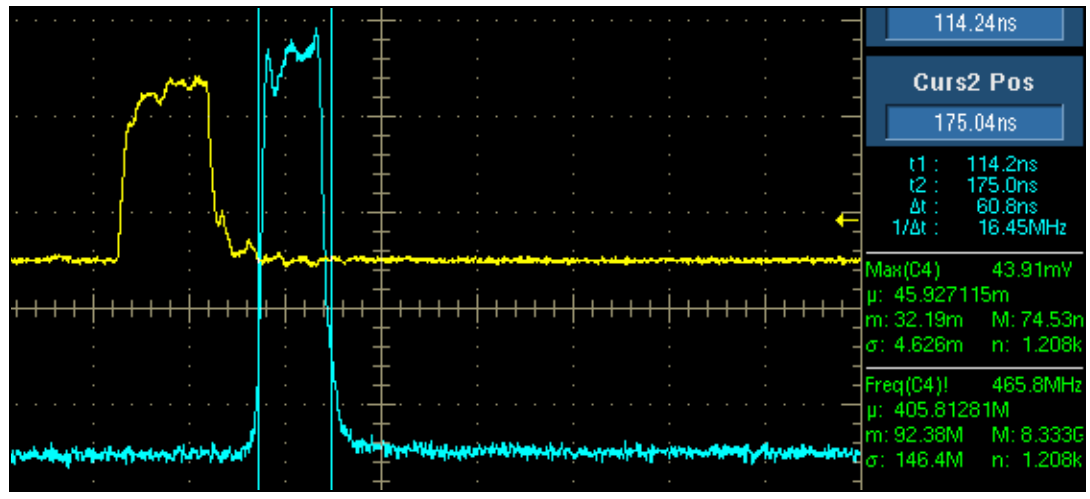

Figure 2: Input signal to AOM (Yellow (upper line): AOM signal, Cyan (lower line): Photodiode reading of deflected pulse)

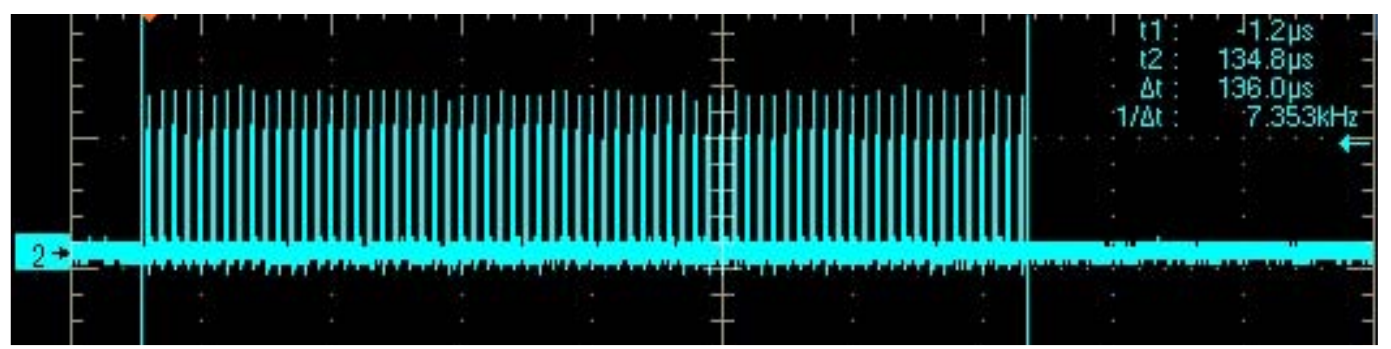

Figure 3: Close view of a single 68-pulse train.

The use of an Acousto-Optic Modulator to deflect pulses is an advancement of the laser system from second-generation designs, which utilize the electro-optic effect using Pockels Cells ${ }^{4}$. While systems using this approach can generate pulses of $<5$ ns duration, limited by the speed of the electric switch, they also require highvoltage, high-current power supplies and control electronics, putting them out of the price range of many 
laboratories. The AOM represents a tenfold decrease in cost with performance limited only by the speed of sound through the crystal and the ability to focus laser light to a sufficiently small point. Pulses of 20 ns duration have been achieved, and with more aggressive focusing within the crystal to decrease transit time of the acoustic wave across the beam diameter, shorter pulses are possible.

These deflected pulses are collimated and passed through an aperture, which prevents undeflected continuous-wave laser light from entering the amplifier chain. Figure 4 shows these various elements as they are installed in the system. A schematic can be seen in Figure 5, which shows the placement of the various optical components.

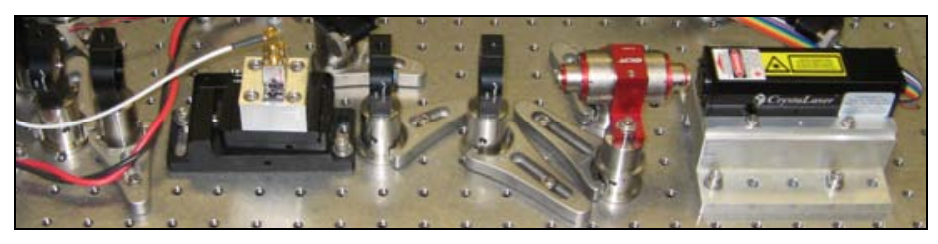

Figure 4: Photograph of Pulse-Slicing System

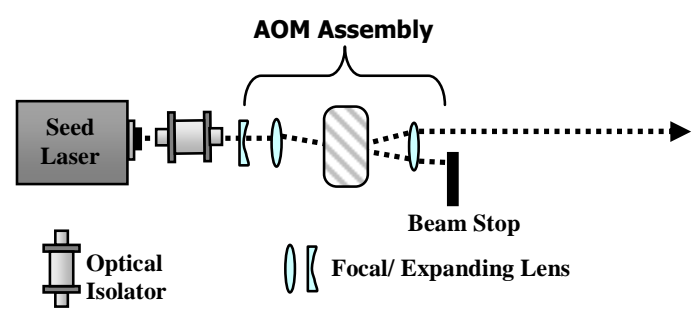

Figure 5: Pulse-Slicing System Block Diagram

\section{B. Pulse Amplification}

For sufficient visible laser output, the low energy input pulses need to be increased to energies in the $\mathrm{mJ} / \mathrm{pulse}$ range. For this purpose, a chain of laser amplifiers is used.

\section{Amplification Theory}

The operation of solid-state laser amplifiers is based on the principles of radiation absorption, spontaneous emission, and stimulated emission within a gain medium. Each amplifier consists of two primary components: a xenon flashlamp and a neodymium-doped yttrium aluminum garnet (Nd:YAG) crystal acting as the gain medium. Nd:YAG crystals have been in use for laser amplification since the 1960s, due to their good optical quality, high thermal conductivity (for reducing thermo-optic effects in pulsed systems), low lasing threshold and high gain.

$\mathrm{Nd}$ :YAG exhibits a four-level energy system, whereby a large population inversion is easy to achieve. This supports a comparatively low lasing threshold and large gain. A drawback to lasing mediums with high gains is the greater probability of amplified spontaneous emission (ASE). ASE occurs due to spontaneous decay from the excited state. This process emits incoherent photons that then are amplified by the rest of the amplifier chain. ASE particularly becomes a problem as pump power is increased. In multi-stage amplification systems, small amounts of ASE can lead to parasitic oscillations that deplete the upper energy levels of the amplifier crystals and significantly reduce output power.

A critical element for reducing parasitic losses due to ASE is the use of Faraday isolators, optical components that act as a one-way valve for light. This is achieved by the use of two polarizers rotated at $45^{\circ}$ to each other, and between them a Faraday rotator. This rotator employs the magneto-optic effect by using a large magnet in combination with an optical substrate to rotate a beam of light while preserving its linear polarization. This rotation is the same regardless of the propagation direction of light. Therefore, light traveling in one direction will be rotated and able to pass through both polarizers, while light traveling the other direction will be rotated, but not in correct alignment to pass through the first polarizer.

\section{Amplification Configuration}

In the pulse burst laser system, three flashlamp-pumped Nd:YAG laser amplifiers are used in a double-pass configuration. As shown in Figure 7, light enters the amplifier for a single pass, and exits the amplifier, where it is then circularly polarized by a $\lambda / 4$ wave plate. This light is then reflected by a $1064 \mathrm{~nm}$ HR-coated mirror, back through the quarter wave plate, resulting in a polarization shift of $90^{\circ}$. The light then enters the amplifier for another single pass, and due to the polarization shift, reflects off of a beam-splitter cube to enter the next amplifier stage. This basic configuration is used for all three stages.

Thermal gradients experienced due to pulsed flashlamp operation cause radial changes in the index of refraction of the Nd:YAG crystal. The radial nature of this change increases the speed of light propagation (since $\mathrm{v}=$ $\mathrm{c} / \mathrm{n}$ ) towards the outside of the crystal, making it behave like a weak positive spherical lens. Therefore, between amplifiers 1 and 2, a -50 and +100 focal length lens are used to change the beam size to double the diameter of the first amplifiers output, and allow it to slightly diverge to take into account this thermal lensing. This beam size is 
necessary to realize optimal gain through amplifiers 2 and 3, which have larger diameter crystals. By filling each crystal rod with the laser beam, the maximum amount of energy can be extracted. Before each stage, a Faraday isolator is used to prevent ASE from entering the optical cavity of the seed laser or other amplifier paths.

Figure 6 is a photo of the amplifier chain installed in the pulse burst laser system. The three amplifiers can be seen complete with a set of lenses for telescoping, and various optics allowing double-pass operation. A schematic can be seen in Figure 7, which shows the placement of the various waveplates and optical components.

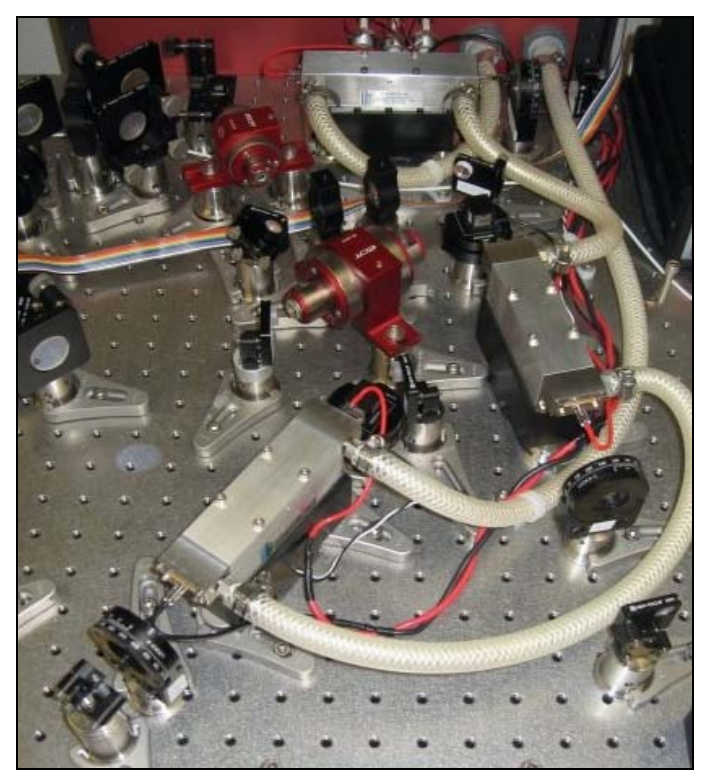

Figure 6: Photo of installed amplifiers and optics allowing double-pass operation

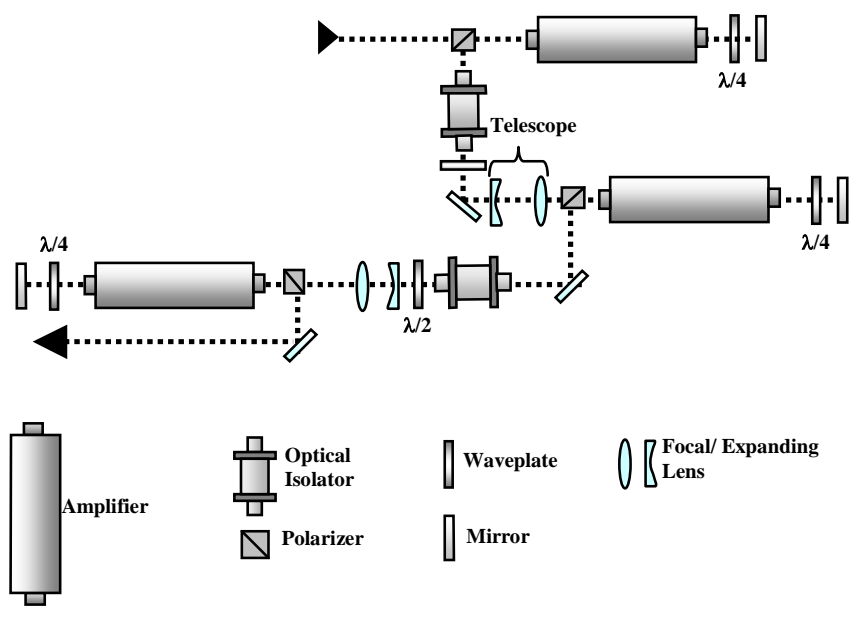

Figure 7: Amplifier Chain Block Diagram, Including Isolators, Mirrors, and Polarizers

\section{Second Harmonic Generation (SHG)}

For flow visualization purposes, the $1064 \mathrm{~nm}$ light generated by the pulse burst laser system must be efficiently converted to a wavelength in the visible spectrum where light scattering by particles is greater and imaging sensors are more sensitive. By using nonlinear optical crystals, visible light can be generated at the second harmonic, for an output beam of $532 \mathrm{~nm}$ (green) light. This technique of generating laser light in the visible wavelength using solid-state lasers has been used in applications ranging from green laser pointers to the large laser systems such as the National Ignition Facility ${ }^{6}$. The theory and setup of frequency conversion is discussed below.

As the fundamental beam propagates, harmonic conversion is continually occurring at all points in the crystal; however, the indices of refraction for light at $1064 \mathrm{~nm}$ and $532 \mathrm{~nm}$ are different, forcing the 532 $\mathrm{nm}$ light to propagate at a slower speed through the crystal. Therefore, at each point, light at $532 \mathrm{~nm}$ is being created, but with a sight difference in phase. These changes can effectively nullify the harmonic beam by destructive interference. Phase matching then refers to using the optical properties of the crystal, particularly the different refractive indices along the crystal's $\mathrm{x}-, \mathrm{y}-$, and $\mathrm{z}-$ axes, to tune the speed of $532 \mathrm{~nm}$ propagation. By changing the speed of propagation, the phases begin to correlate, allowing constructive interference to increase the amplitude of the harmonic beam. For KTP, this phase matching angle is 23 degrees. To achieve this angle, the KTP crystal is locked on to a tunable stage, the output is reflected from two $532 \mathrm{~nm}$ HR-coated mirrors, and is recorded using a thermal optical power meter. By tuning to the highest power output with constant laser parameters of the fundamental beam, this optimal phase matching angle is empirically reached. The tunable stage and $\lambda / 2$ wave plate are shown in Figure 8.

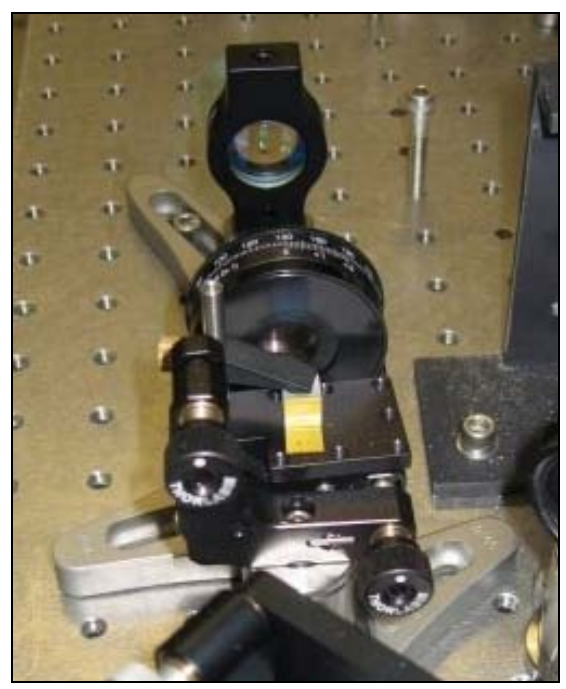

Figure 8: Close-up of $\lambda / 2$ wave plate and KTP crystal mounted on a tunable stage. 


\section{Amplification / SHG Findings and Results}

Single-pulse measurements are the first indication of system performance. They also indicate the usefulness of the system for traditional flow illumination and use in a particle image velocimetry (PIV) system. The output power of longer duration pulses, from $1 \mu \mathrm{s}$ to $25 \mu \mathrm{s}$, and shorter duration pulses, from $50 \mathrm{~ns}$ to $750 \mathrm{~ns}$ is plotted in Figures 9 and 10, respectively:

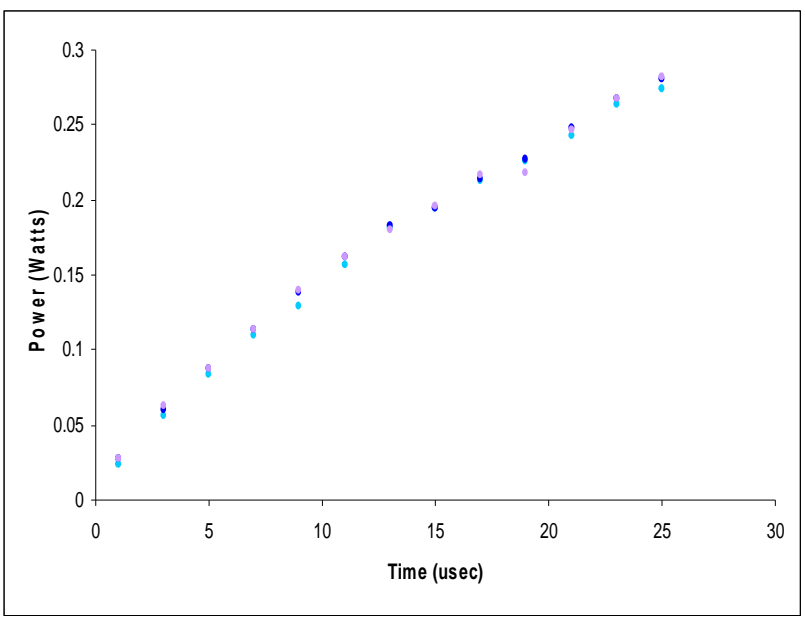

Figure 9: Single Long-Duration Fundamental Pulse Power, $500 \mu$ s Delay, $5 \mathrm{~Hz}$

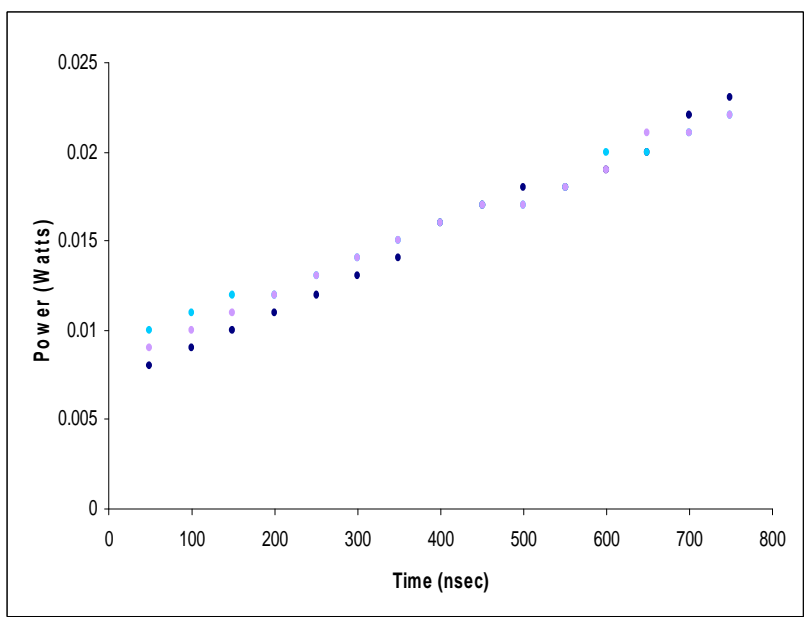

Figure 10: Single Short-Duration Fundamental Pulse Power, $500 \mu$ s Delay, 5 Hz

An approximately linear relationship is found at these pulse durations, useful for low to moderate speed flow illumination.

For 3-D imaging, 68 high-energy pulses are created within a total window of 136 us. This window does not change, but the individual pulse durations can be varied, with a subsequent decrease in the idle time between pulses. Figure 11 illustrates the power achieved by varying this high time. The sharper decrease around $25 \mathrm{~ns}$ is caused by the inability of the AOM to shape pulses shorter than 30 ns using the current focusing scheme.

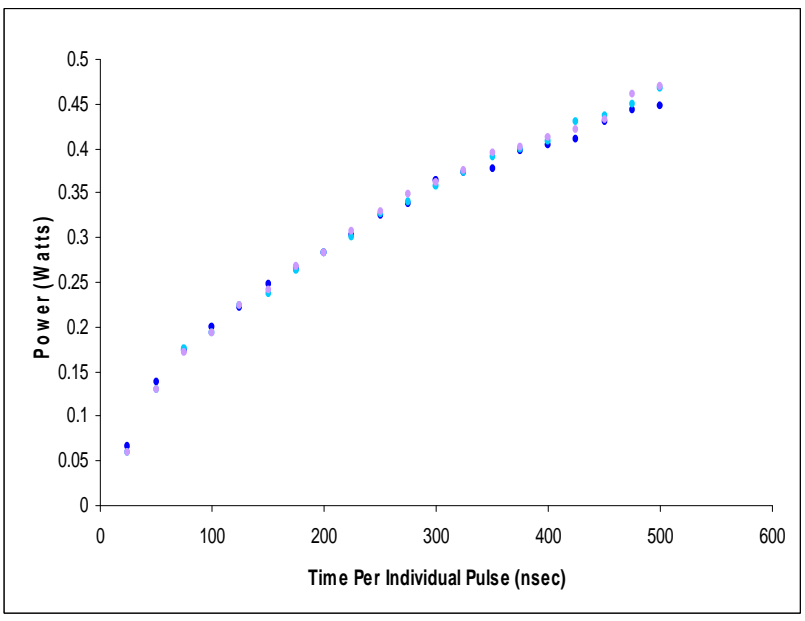

Figure 11: 68-Pulse Train Fundamental Power, $500 \mu \mathrm{s}$ Delay, $5 \mathrm{~Hz}$

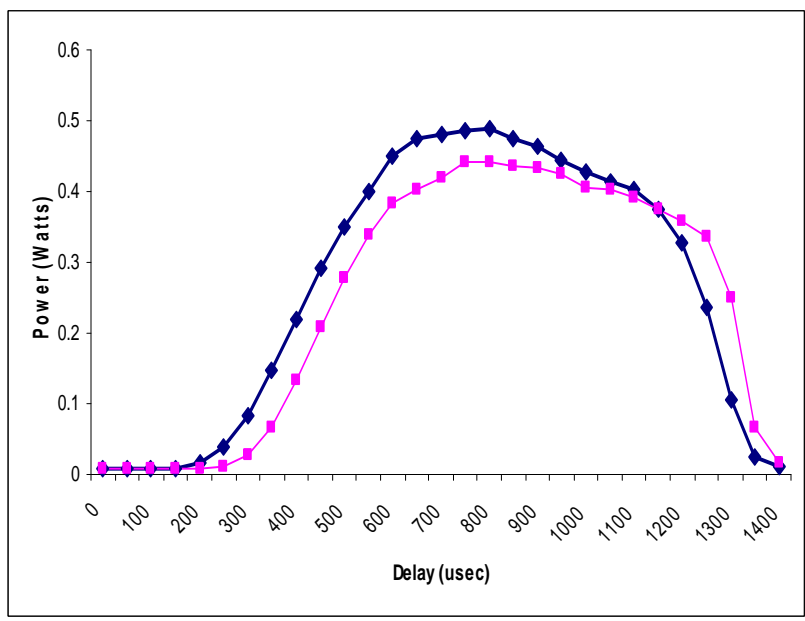

Figure 12: Effect of Delay on Fundamental Output Power (Blue: 300 ns 68-pulse train, Pink: 25us Single Pulse)

SHG efficiencies of 35\% have been obtained in these cases using tight focusing through the crystal. In addition to using the pulse duration to achieve maximum power, the delay of the laser pulses can be varied to propagate through the crystals at their most excited state. The state of the crystals is directly related to the luminosity 
profile of the flashlamps. Figure 12 shows the power output by using the same input laser, simply at different delays. These power profiles very closely correlate with the luminosity profile of the flashlamps.

The maximum power is produced in a range from $700 \mu \mathrm{s}$ to $900 \mu \mathrm{s}$ after the beginning of the flashlamp firing. For applications using single pulses, delays in this range can be used. However, if multiple pulses are required, the pulse must be repositioned earlier in the flash. This prevents a buildup of excited atoms that are stimulated to a ground state in the first few pulses in a pulse train. Placing the pulses within the portion of the luminosity profile with a positive slope creates an approximate equilibrium where the number of excited atoms and number of stimulated atoms remains relatively constant, resulting in constant energy for each pulse in a multiplepulse train. This equilibrium condition occurs at a delay of $475 \mu$ s for a 68-pulse train of $300 \mathrm{~ns}$ pulses. Another useful performance measurement is the effect of the repetition rate on the output power. A linear relationship exists as the flashlamps are fired from $1-8 \mathrm{~Hz}$, so the per-pulse energies are approximately the same. Therefore, the laser can be used in applications where various repetition rates may be desired.

\section{Component Synchronization and Calibration}

The high-speed three-dimensional imaging concept is achieved by deflecting a sheet of $532 \mathrm{~nm}$ light and taking images as the laser sheet sweeps through the flow. For this technique to work properly, synchronization and calibration of the AOM modulator, laser amplifiers, galvanometric scanning mirror, and high-speed camera is needed.

\section{A. Laser Triggering}

The flashlamp power supply provides the initial trigger for all components of the system, and can be varied in pulse width from $100 \mu \mathrm{s}$ to $1 \mathrm{~ms}$, and frequency from 1 to $10 \mathrm{~Hz}$. This trigger is sent to a computer as an input to a National Instruments NI-6602 Counter-Timer card. Using Labview, this signal from the power supply triggers another, "Holdoff," trigger signal. This provides a noise-free signal that can be routed within the computer, and prevents additional triggers due to the large amount of noise generated in the power supply trigger due to its internal high voltage components. The "Holdoff" trigger signal is used to trigger a "Delay" trigger. The amount of delay between the "Holdoff" and "Delay" triggers can be varied in Labview. This "Delay" trigger then triggers the 68pulse train that is output to the AOM. Figure 13 shows the relationship of signals between system components.

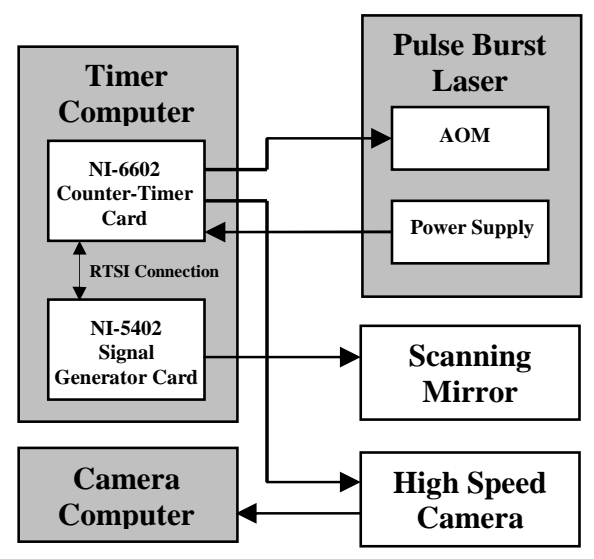

Figure 13: Relationship of Signals Between System Components

This multitude of triggers is used to isolate noise sources as much as possible and allow for high configurability. This configurability is critical for calibration of each of the 68 laser pulses, which must have approximately the same amount of energy contained within each to achieve accurate flow visualization. By varying the delay, the 68-pulse train can be varied in position to the flashlamp firing. This results in approximately constant depletion and excitement rates within the Nd:YAG amplifier crystals, and thus constant amplification of each pulse. 


\section{B. Scanning Mirror}

For deflection of light across the flow field, a GSI Lumonics VM500 galvanometric scanning mirror is used. Characterization of its performance has been documented in Satija and Thurow ${ }^{7}$, where an angular velocity of over $100,000 \mathrm{deg} / \mathrm{sec}$ is reported given a step command voltage, rendering it sufficient for the high deflection speeds needed as part of a three-dimensional imaging system. To incorporate this device, a step signal is output using an NI-5402 signal generator card that is triggered from the internally routed Holdoff trigger mentioned previously. By inputting the pulsewidth of the power supply trigger, the LabView control program calculates the signal frequency to generate one deflection per pulse. The total signal sent to the mirror is longer than the power supply pulsewidth to allow the mirror adequate time to return to its starting position and ensure consistent operation between pulses.

The timing and amplitude of the step signal are critical for correct calibration of the mirror. The amplitude of the step is responsible for the magnitude and speed (slope) of the deflection, which is directly proportional to the change $\Delta \theta$ between pulses. This allows the total width of the deflected sheets to be changed in order to fit the application. The step must be timed to position the laser pulse train in an approximately linear portion of the deflection envelope, so each pulse is deflected with approximately equal $\Delta \theta$. By adjusting the starting phase of the output signal, this can be precisely controlled for a particular step amplitude, as the deflection characteristics change depending on amplitude. Figure 14 demonstrates the correct positioning where the input to the mirror calls for a deflection of $\sim 10$ degrees.

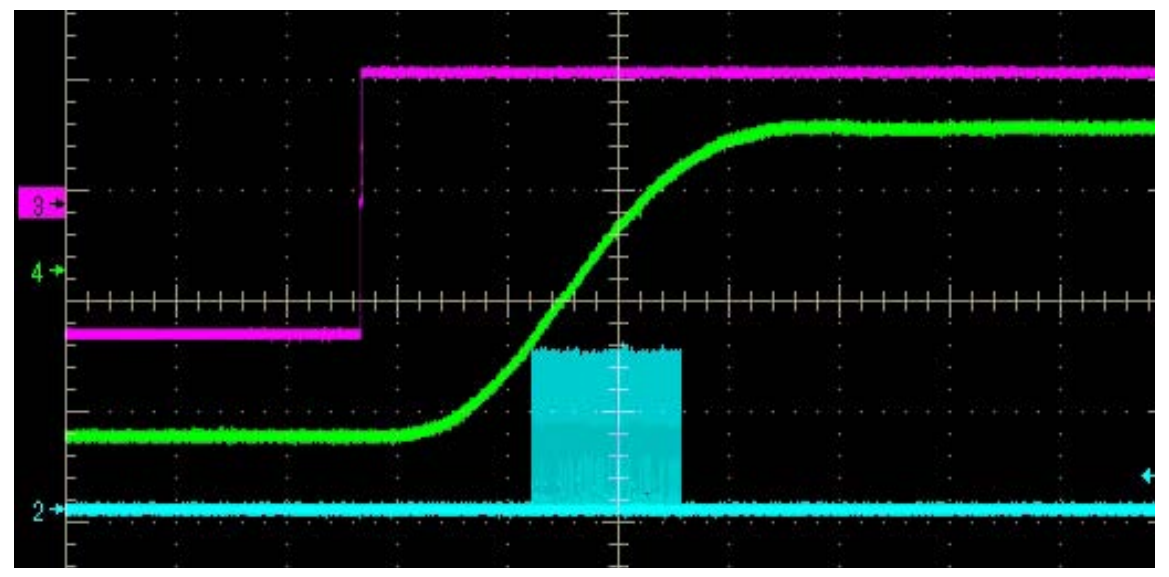

Figure 14: Timing of Linear Portion of Deflection with Laser Pulses (Purple: Step Signal, Green: Deflection Indicator, Cyan: Laser Pulses)

\section{High-Speed Camera}

A DRS Hadland Ultra68 camera is used to capture images of the moving laser sheet illuminating the flow. The camera is capable of capturing 500,000 frames per second with exposure times from $10 \mathrm{~ns}$ to a full $2 \mu \mathrm{s}$. Dedicated software from the manufacturer controls all aspects of the camera, including triggering and exposure sequence. To begin the trigger sequence, the Holdoff trigger signal is output to the camera. Using the camera software, the delay is input minus the manufacturer reported internal camera delay of $85 \mathrm{~ns}$. The actual exposure timing of the camera is output using the camera's external monitor feature. Using an oscilloscope with the monitor signal input and a photodiode input of laser output, fine adjustments can be made to the camera delay to position the exposures to capture each laser pulse and adjust the exposure time accordingly 


\section{3-D Image Reconstruction}

The DRS Hadland Ultra68 outputs all 68 images in bitmap format. 8 pictures out of a 68 image sequence of an acoustically excited jet are shown in Figure 15. The frequency of the speaker in the flow facility was modulated to create a stable ring vortex structure in the camera's field of view. The output images contain slight amounts of noise, and certain distortions due to the operation of the camera. The Ultra68 uses a beamsplitter to send light entering the aperture to a four-quadrant CCD chip. High-speed operation at 500,000 fps is then achieved by running each quadrant at an effective speed of 125,000 fps. The limiting factor in this configuration is the buffer, which allows the CCD to store a maximum of 68 images before the data must be read out. This method of operation introduces small distortions, so MATLAB is used to condition this data and apply transformations, and Tecplot uses this data to render detailed visuals.

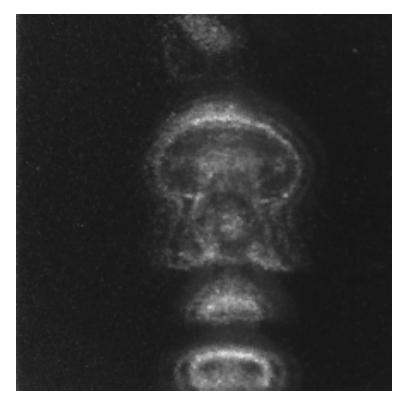

$\mathrm{t}=32 \mu \mathrm{s}$ $\mathrm{Z}=1.20 \mathrm{~cm}$

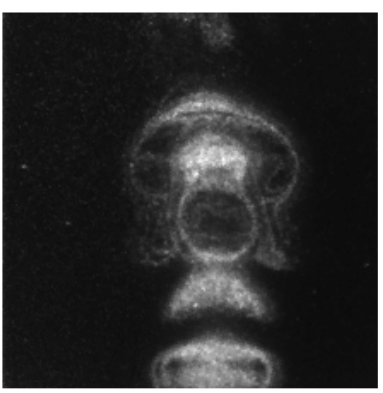

$\mathrm{t}=40 \mu \mathrm{s}$ $\mathrm{Z}=1.49 \mathrm{~cm}$

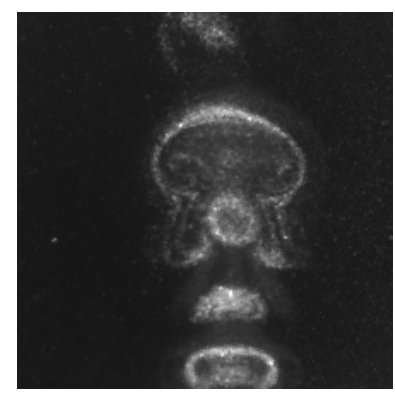

$\mathrm{t}=34 \mu \mathrm{s}$

$\mathrm{Z}=1.27 \mathrm{~cm}$

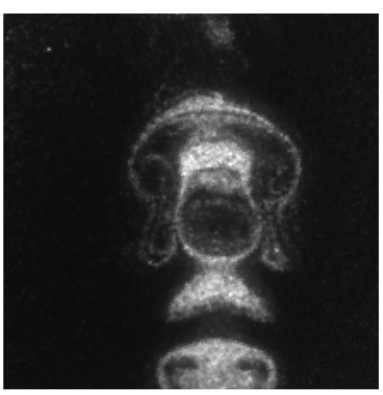

$\mathrm{t}=42 \mu \mathrm{s}$

$\mathrm{Z}=1.57 \mathrm{~cm}$

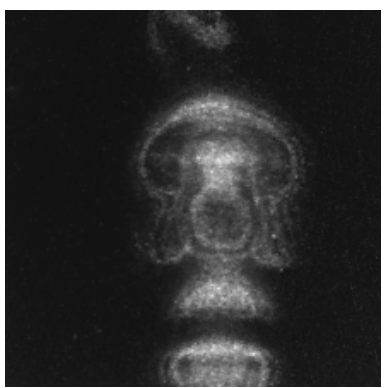

$\mathrm{t}=36 \mu \mathrm{s}$

$\mathrm{Z}=1.34 \mathrm{~cm}$

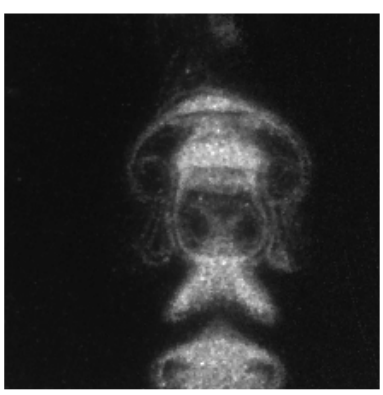

$\mathrm{t}=44 \mu \mathrm{s}$

$\mathrm{Z}=1.64 \mathrm{~cm}$

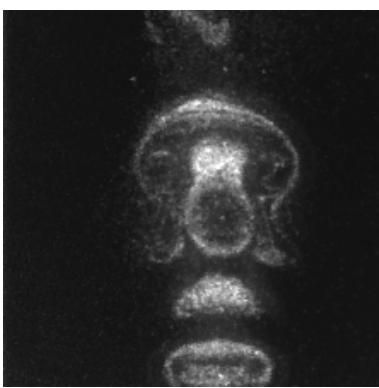

$\mathrm{t}=38 \mu \mathrm{s}$

$\mathrm{Z}=1.42 \mathrm{~cm}$

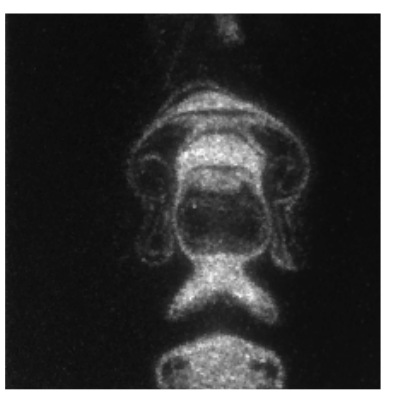

$\mathrm{t}=46 \mu \mathrm{s}$

$\mathrm{Z}=1.72 \mathrm{~cm}$

Figure 15: Raw Images 16 through 23 from a 68-Image Sequence

\section{A. MATLAB Conditioning}

MATLAB is used to apply a dark image subtraction to eliminate ambient light created from the flashlamp firing, and any small changes in CCD sensitivity. A dark image sequence is taken with the flashlamps firing, but with the seed laser's aperture closed. The actual image sequence is then taken, and each picture of the actual image has its corresponding dark image subtracted from it. After this correction, a spatial transformation is performed using the imtransform command on each individual image. Calibration is done using a dot card placed in the area to be imaged. By knowing the actual pattern imprinted on the card, MATLAB can create an image transformation matrix. This is important to correct for slight errors that accompany the particular method of operation of the Ultra68 camera, which uses an image intensifier and beamsplitter, both of which can slightly distort the image. When the transformation matrix is applied to the original image, the distortion is greatly reduced; when not applied, the images have a "jitter" across every four images, consistent with the 4-way beamsplitter integrated into the design of the Ultra68. Finally, after all transformations are applied, the images are exported into .dat ASCII-format files that are compatible with the Tecplot Data Loader. 


\section{B. TecPlot 360 Visualization}

The ASCII output file from MATLAB is then loaded into Tecplot, which automatically performs a conversion from ASCII to binary Tecplot data file. This raw data then needs another correction to properly distance the individual data slices from one another, and correlate axis values to actual distances. By taking a picture of a ruler in the $\mathrm{x}$ and $\mathrm{y}$ orientations using the high speed camera, and measuring the sweep of the laser sheet, the axes can be initialized with proper dimensions and the Z-coordinate can be "stretched" to match the actual width of the laser sheet. Visualizations then have a correct scale, and can be used for scientific study of the flow.

After the dimensional corrections are made, a multitude of visualizations can be configured. Using Tecplot's iso-surface features, points of constant intensity are mapped between all 68 data planes. By changing the intensity value, it is possible to look at both core flow structures and weaker fragments. Figure 16 shows four different image sequences taken using the Acoustically-Excited Jet flow facility at Auburn. By modulating the acoustic frequency, different flow shapes can be easily created. As seen in the top-left and top-right images, the three-dimensional, symmetric structure of ring vortices, and their internal structures can easily be seen using a translucency feature of Tecplot. Being able to capture the entire flow, rather than just a two-dimensional sheet, may also be useful in studies of structures displaying less symmetry, such as in the bottom-left.
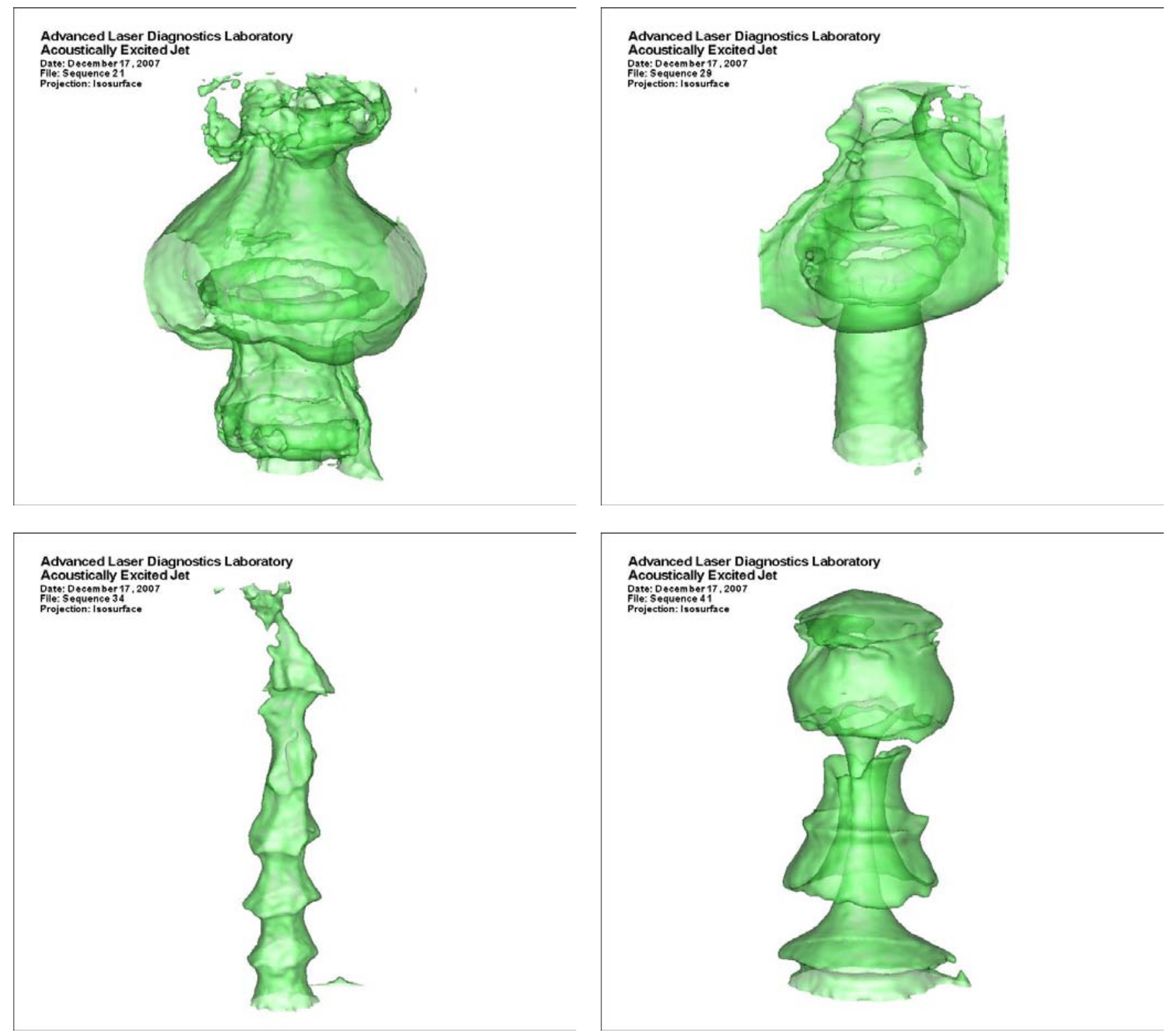

Figure 16: Four iso-surface projections of flows created by the Acoustically Excited Jet Facility 
One great advantage of three-dimensional visualization is the amount of two-dimensional data that can also be extracted. Slices can be taken along the X-, y-, or z- axes. By slicing the data perpendicular to the YZ plane as in Figure 17, the flow can be seen through 68 planes (at $220 \times 220$ pixel resolution) in the laser propagation direction, allowing 2-D pictures similar to traditional visualization techniques. However, slicing the data perpendicular to the $\mathrm{XY}$ axis as in Figure 18 provides an entirely new perspective on the flow, with 220 planes of data (at $68 \mathrm{x} 220$ pixel resolution)
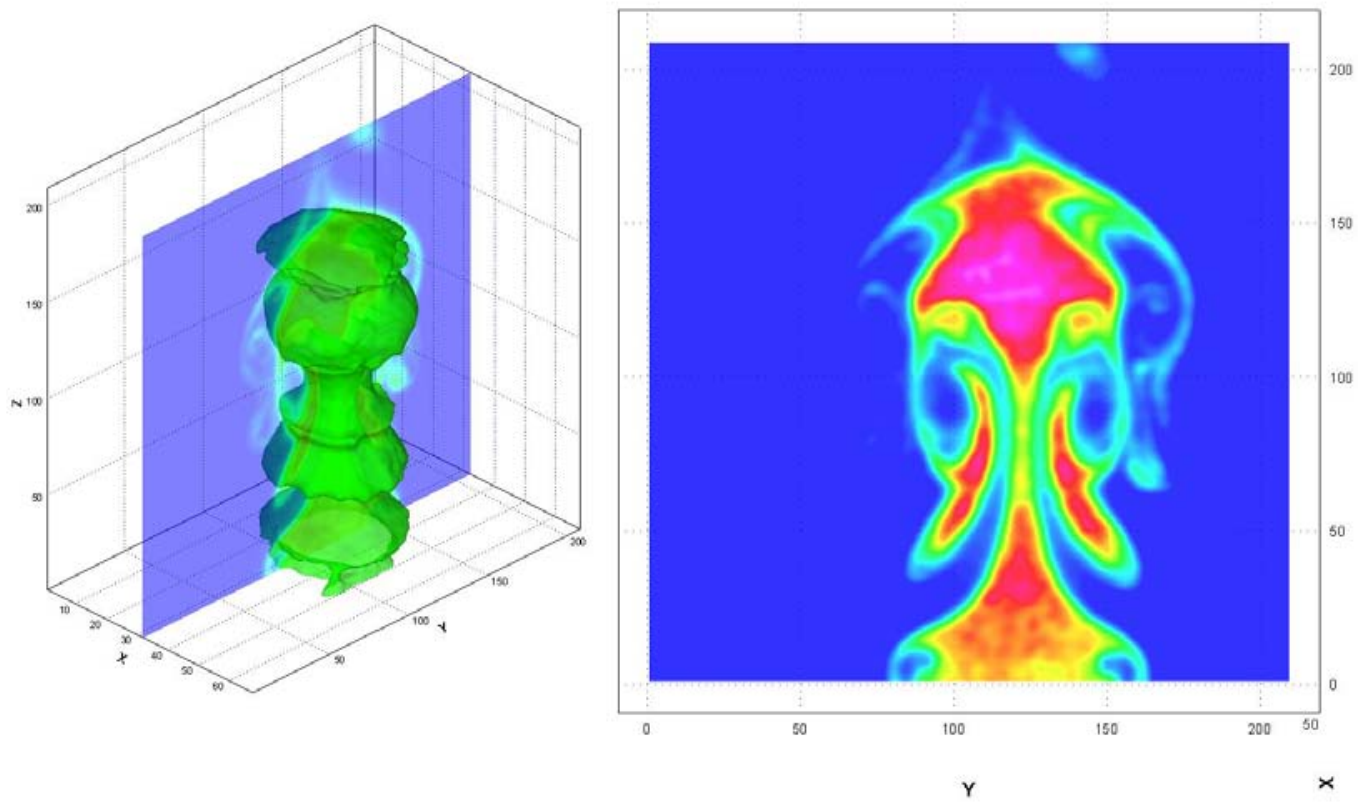

Figure 17: Two-Dimensional Slicing in the YZ Plane
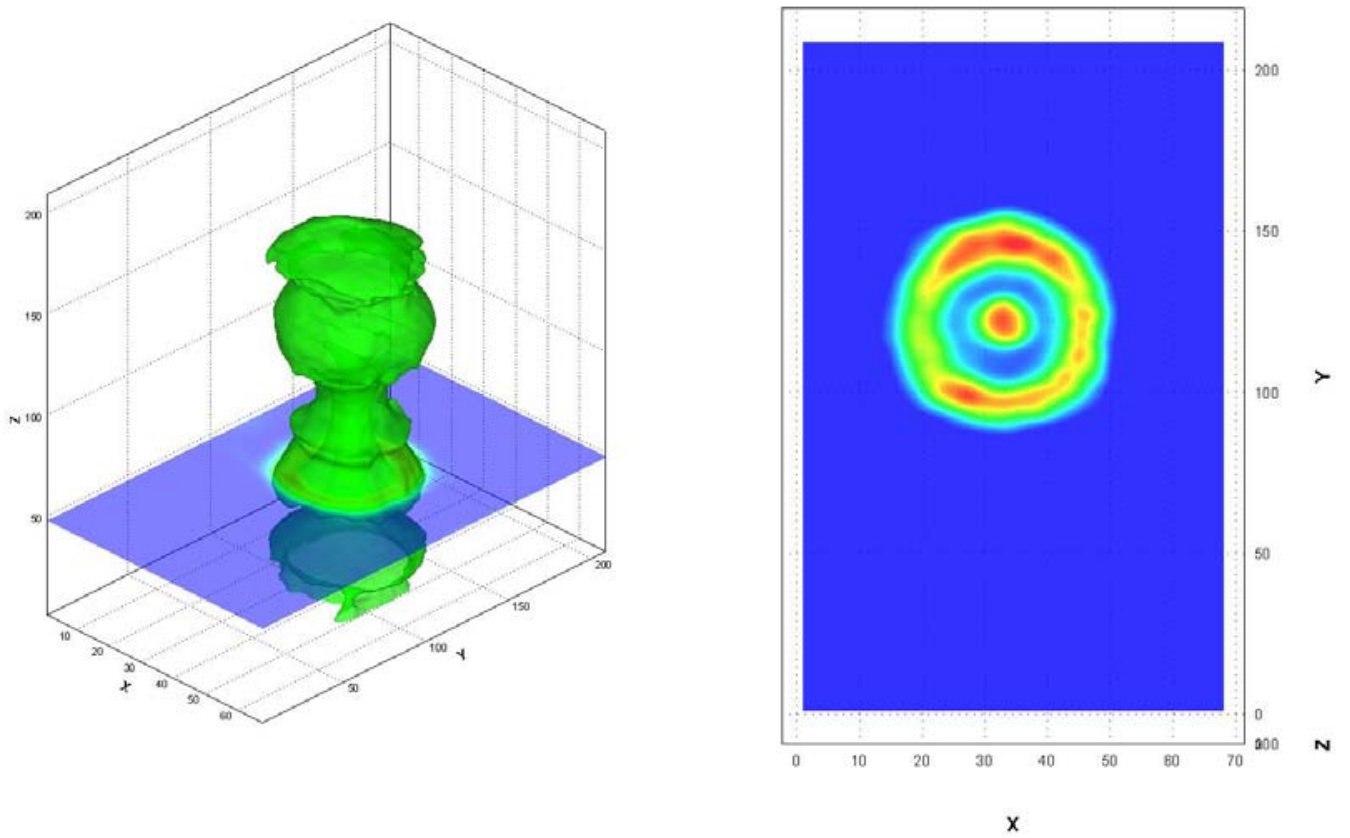

Figure 18: Two-Dimensional Slicing in the XZ Plane

American Institute of Aeronautics and Astronautics 


\section{Conclusions \& Future Work}

The results obtained using the Auburn University Pulse Burst Laser in combination with a high-speed scanning mirror and high-speed camera demonstrate the effectiveness of the scanning technique for threedimensional flow visualization at moderate speeds and across an area whereby 68 planes of data can approximately characterize the flow. By slicing the image perpendicular to the various imaging axes, two-dimensional data can also be obtained at any point of interest in the flow.

In order to be effective for higher flow velocities, more power can be generated at shorter pulse durations allowing for faster exposures, and faster cameras can be used. Work is continuing on the pulse burst laser to increase the fundamental power output by better alignment, beam sizing, and modification of the flashlamp power supply to deliver more pump energy to the amplifiers. Attention is also being paid to SHG processes. Various focusing techniques and the use of more exotic crystals such as periodically-poled KTP (PPKTP) could increase conversion efficiencies above $40 \%$. High-efficiency Fourth-harmonic generation to $266 \mathrm{~nm}$ has been demonstrated at other laboratories by use of $\mathrm{CsLiB}_{6} \mathrm{O}_{10}$ crystal $^{8}$, and a similar conversion is planned to adapt the pulse burst laser for support of acetone fluorescence.

New cameras are now available with performance characteristics exceeding the Ultra68. Some models can take 100 frames of $200 \times 300$ pixel resolution at 1,000,000 fps. This would allow for over 30 new planes of data to be collected in an even shorter amount of time, thus increasing the effective flow velocity that can be captured.

New data analysis techniques are also being explored. Proper orthogonal decomposition (POD) is a tool used for finding the most common and strongest features in turbulent flows by creating a correlation matrix that relates individual images to the rest of the images in a large data set. By finding the eigenvalues of this matrix, unique linear combinations of images, or "modes," can be assembled, each defining more subtle flow features. This capability has already been demonstrated on data sets of 2D images, and is currently being modified to suit a data set of 3D images taken of various flow facilities.

\section{Acknowledgments}

The support of the Army Research Office through the Young Investigator Program is gratefully acknowledged. The first author would like to thank David Wall, Bobby Thompson, and Adam Willis for their work in creating flow facilities for study and their help in conducting experiments. The help of Aman Satija with alignment and system design is also appreciated.

\section{References}

1 Patrie, B.J., Seitzman, J.M., and Hanson, R.K., "Instantaneous Three-Dimensional Flow Visualization by Rapid Acquisition of Multiple Planar Flow Images,” Opt. Eng., vol. 33, pp. 975-980, 1994.

2 Lempert, W. R., Wu., P., Zhang, B., Miles, R. B., Lowrance, J. L., Mastracola, V., and Kosonocky, W. F., "Pulseburst laser system for high speed flow diagnostics," AIAA Paper 96-0179, 1996.

$3 \mathrm{~W} \mathrm{u}$, P., Lempert, W. R., and Miles, R. B., "MHz pulse-burst laser system and visualization of shockwave/boundary-layer interaction in a Mach 2.5 wind tunnel," AIAA J., Vol. 38, 672, 2000.

4 Thurow, B., Hileman, J., Samimy, M., and Lempert, W., “A MHz Rate Imaging System for Study of Turbulent and Time Evolving High Speed Flows," proceedings of the 19th International Congress on Instrumentation in Aerospace Simulation Facilities, Cleveland, OH, August 2001.

5 Thurow, B. and Satija, A., "Design of a MHz Repetition Rate Pulse Burst Laser System at Auburn University," AIAA Paper 2006-1384, 44th AIAA Aerospace Sciences Meeting, Reno, NV, 2006.

6 Lawrence Livermore National Laboratory, "National Ignition Facility laser performance status,” Applied Optics, vol. 46, pg 3286-3287, 2007.

7 Thurow, B., and Satija, A., "Further Development of a High-Speed Three-Dimensional Flow Visualization System," AIAA Paper 2007-1060, 45th AIAA Aerospace Sciences Meeting, Reno, NV, 2007.

8 Yap, Y., Inagaki, M., Nakajima, S., Mori, Y., and Sasaki, T. "High-Power fourth- and fifth-harmonic generation of a Nd:YAG laser by means of $\mathrm{C} \mathrm{CsLiB}_{6} \mathrm{O}_{10}$ " Optics Letters, vol. 21, pp 1348-1350, 1996. 